\title{
Phenethyl Isothiocyanate Inhibits Nasopharyngeal Carcinoma Cell Growth and Invasion
}

\author{
Chenggang Mao ${ }^{1}$, Xiaochun Zhou ${ }^{1}$, Yidao Jiang ${ }^{1, ~}$, Lijia Wan ${ }^{1}$, Zezhang Tao ${ }^{2}$ \\ ${ }^{1}$ Department of Otolaryngology-Head and Neck Surgery, Jingzhou Central Hospital, The Second Clinical Medical College, Yangtze \\ University, Jinzhou, China \\ ${ }^{2}$ Department of Otolaryngology-Head and Neck Surgery, Renmin Hospital of Wuhan University, Wuhan, China
}

\author{
Email address: \\ 22101163@qq.com (Yidao Jiang) \\ ${ }^{*}$ Corresponding author
}

\section{To cite this article:}

Chenggang Mao, Xiaochun Zhou, Yidao Jiang, Lijia Wan, Zezhang Tao. Phenethyl Isothiocyanate Inhibits Nasopharyngeal Carcinoma Cell Growth and Invasion. Journal of Diseases and Medicinal Plants. Vol. 5, No. 1, 2019, pp. 10-16. doi: 10.11648/j.jdmp.20190501.12

Received: January 4, 2019; Accepted: January 23, 2019; Published: February 15, 2019

\begin{abstract}
The dietary compound phenethyl isothiocyanate (PEITC), is an active component of cruciferous vegetables and markedly inhibits the growth of a variety of tumors. However, its role in human nasopharyngeal carcinoma (NPC) is obscure. The aim of the present study was to elucidate the possible mechanisms whereby PEITC exhibited anticancer properties in human nasopharyngeal carcinoma NPC-TW01 cells in vitro. The experiment results exhibited that in a dose- and time-dependent manner treatment of NPC-TW01 cells with PEITC significantly inhibited cell proliferation, promoted apoptosis with concurrent G2/M cell cycle arrest and inhibited cell invasion in a dose-dependent manner. These effects were accompanied by significant alterations in the expression levels of key proteins associated with pro-survival signaling pathways, including PI3K, Akt, ERK, NF- $\mathrm{B}, \mathrm{Bcl}$, Bax, cyclin B, CDK4 and CDK6. Significantly, these effects were not reflected in $16 \mathrm{HBE}$ normal human bronchial epithelial cells, indicating a safe range of treatment concentrations between 0 and $10 \mu \mathrm{M}$ PEITC. In conclusion, PEITC exhibited significant anticancer effects against human nasopharyngeal carcinoma cells in vitro with low toxicological impact on normal bronchial epithelial cells. This was achieved through dysregulation of key proteins involved in the occurrence and development of tumors, and this approach may be applied to the clinical treatment of NPC and in drug screening.
\end{abstract}

Keywords: Phenethyl Isothiocyanate, Nasopharyngeal Carcinoma, NPC-TW01, Anticancer, Toxicity

\section{Introduction}

In nasopharyngeal carcinoma (NPC), tumors originate from the epithelial cells that cover the surface of the nasopharynx. The incidence of NPC is particularly high in Chinese and Tunisian populations [1]. At diagnosis, $70 \%$ of patients have locally advanced, non-metastatic stage III or IV NPC [2, 3]. Despite novel advances in radiotherapy, chemotherapy and gene-targeting agents, the overall survival rate of patients with aggressive phase NPC remains low [4].

Although a number of chemotherapeutic drugs are available for the treatment of cancer, a highly effective and less toxic approach for treating NPC is required. One potential resource for a new generation of therapeutic agents targeting the prevention and treatment of NPC may be natural substances. Epidemiological and case-control studies have indicated a strong correlation between the consumption of certain vegetables and the decreased risk of carcinogenesis $[5,6]$. Recently, it has been suggested that phenethyl isothiocyanate (PEITC), an important tumoricidal component found in cruciferous vegetables such as broccoli and cauliflower may possess anticancer properties against various malignancies, including breast, colon and prostate cancers [7-11]. Several mechanisms have been proposed for these actions, including the generation of reactive oxygen species and initiation of cell cycle arrest [12]. However, the role of PEITC in human nasopharyngeal carcinoma cells remains largely unknown.

The primary aim of the study was to determine the actions and potential mechanisms of PEITC in nasopharyngeal carcinoma, by studying its effects on proliferation, apoptosis, cell cycle and metastasis in human nasopharyngeal carcinoma 
NPC-TW01 and normal bronchial epithelial 16HBE cells in vitro in order to provide a basis for targeted therapies and drug screening in patients with NPC.

\section{Materials and Methods}

\subsection{Cell lines, Reagents and Kits}

Human nasopharyngeal carcinoma cell line NPC-TW01 and human normal bronchial epithelial cell line 16HBE were cryopreserved in our laboratory and stored in liquid nitrogen. PEITC was obtained from Sigma-Aldrich (St. Louis, MO, USA). Other reagents included dimethyl sulfoxide (DMSO) (Sigma-Aldrich), fetal bovine serum (FBS; HyClone, Logan, UT, USA), RPMI-1640 medium and $0.25 \%$ trypsin solution (Invitrogen, Carlsbad, CA, USA). Experimental equipment included Cell Counting Kit-8 (CCK8; Dongji, Japan), Annexin V-FITC/propidium iodide (PI) apoptosis detection kit, PI cell cycle analysis kit (both from Lianke, China), TUNEL apoptosis detection kit (Roche, Indianapolis, IN, USA) and a Transwell insert chamber coated with Matrigel (BD Biosciences, San Jose, CA, USA). Primary antibodies against Bcl-2, Bax, Bcl-xl, PI3K class III, PI3K p110 $\alpha$, PI3K

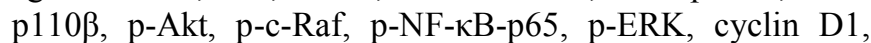
CDK4 and CDK6 and GAPDH were all purchased from Cell Signaling Technology (Danvers, MA, USA).

\subsection{Cell Culture and Treatments}

NPC-TW01 and 16HBE cells were cultured in RPMI-1640 medium supplemented with $10 \%$ FBS and $20 \mu \mathrm{g} / \mathrm{ml}$ antibiotics (ampicillin and kanamycin) at $37^{\circ} \mathrm{C}$ in a humidified atmosphere of $5 \% \mathrm{CO}_{2}$. The cells were harvested in their logarithmic growth phase by trypsinization for use in the experiments. The cells were seeded in 96-well plates at a density of $1 \times 10^{3}$ cells/well for normal culture. They were divided into groups in 6-well plates and treated by adding PEITC to give the following final concentrations: NPC-TW01 cells, 0, 2.5, 5, 7.5 and $10 \mu \mathrm{M}$; and 16HBE cells, 0, 5, 10, 15 and $20 \mu \mathrm{M}$. All experiments were performed at least three times.

\subsection{Cell Proliferation Assay}

NPC-TW01 and 16HBE cells were treated with PEITC as described above, for $0,24,48$ and $72 \mathrm{~h}$ before being incubated with $10 \mu \mathrm{lCCK}-8$ for $1 \mathrm{~h}$ at $37^{\circ} \mathrm{C}$. DMSO was used as a negative control. Six repeats were prepared for each treatment group. Absorbances were detected at $450 \mathrm{~nm}$, and IC50 values were calculated by sigmoidal dose-response nonlinear regression analysis using GraphPad Prism software version 5.04 (GraphPad Software, San Diego, CA, USA).

\subsection{Flow Cytometry with Annexin V-FITC for the Detection of Apoptosis}

After being treated with PEITC for $24 \mathrm{~h}$ as described above, the cells were harvested by trypsinization, centrifuged and washed in cold phosphate-buffered saline (PBS). The cells were then stained with $5 \mu$ Annexin V-FITC solution and 10 $\mu \mathrm{l}$ of PI solution for $15 \mathrm{~min}$. Stained cells were analyzed using a FACSCanto ${ }^{\text {TM }}$ II spectrometer (BD Biosciences). Data were analyzed using FlowJo version 7.6.5 software (FlowJo LLC, Ashland, OR, USA).

\subsection{Flow Cytometry with PI for Cell Cycle Analysis}

After treatment with PEITC for $24 \mathrm{~h}$ as described above, the cells were fixed in $70 \%$ ethanol overnight. The cells were centrifuged and the cell pellets were recovered and resuspended in $1 \mathrm{mg} / \mathrm{ml}$ RNase and $20 \mu 10.5 \%$ Triton X-100. The cells were then incubated with $5 \mu \mathrm{l}$ of $1 \mathrm{mg} / \mathrm{ml}$ PI solution for $30 \mathrm{~min}$ at room temperature. Cell cycle distribution was analyzed using FlowJo software, and the percentages of cells at each phase of the cell cycle were calculated.

\subsection{TUNEL Assay for Detection of Apoptosis}

NPC-TW01 cells were treated with PEITC for $24 \mathrm{~h}$ as described above, and then washed in PBS, air dried and fixed with freshly prepared $4 \%$ paraformaldehyde. Terminal deoxynucleotidyl transferased UTP nick end labeling (TUNEL) was performed according to the manufacturer's protocol (Roche). In brief, the cells were incubated with TUNEL reaction mixture for $1 \mathrm{~h}$ at $37^{\circ} \mathrm{C}$. The slides were washed in PBS and stained with 4',6-diamidino-2-phenylindole (DAPI) before being viewed under microscopy. Six fields were randomly selected from every sample, and 100 cells were randomly selected from every field. The apoptotic rate was calculated as the total number of apoptotic cells $/ 100 \times 100 \%$.

\subsection{Transwell Invasion Assay}

Cell invasion assays were performed using Transwell migration chambers with Matrigel-coated inserts (BD Biosciences) according to the manufacturer's protocol. In brief, $1 \times 10^{5} \mathrm{CNE} 2$ cells were suspended in $200 \mathrm{ml}$ serum-free RPMI-1640 medium and treated wit PEITC for $48 \mathrm{~h}$ as described above. The cells were seeded in Matrigel-coated inserts in the upper chamber; the lower chamber contained RPMI-1640 medium with 10\% FBS as the chemoattractant. After incubation for $48 \mathrm{~h}$ at $37^{\circ} \mathrm{C}$ in a humidified atmosphere of $5 \% \mathrm{CO}_{2}$, any cells that had not penetrated the membrane were removed using cotton swabs; the cells that had successfully migrated to the bottom surfaces of the membranes were fixed with $4 \%$ polyoxymethylene and stained with $0.1 \%$ crystal violet for $20 \mathrm{~min}$. They were counted under a microscope at a magnification of $\times 100$.

\subsection{Western Blotting}

NPC-TW01 cells were treated with PEITC for $24 \mathrm{~h}$ as described above. Total cell lysates were extracted from the harvested cells using complete protease inhibitor 'cocktail'(Roche) and $2 \mathrm{mM}$ dithiothreitol (DTT). The proteins were resolved by $12 \%$ SDS-PAGE and transferred to polyvinylidene fluoride (PVDF) membranes before 
being incubated with the following primary antibodies: anti-Bcl-2, anti-Bax, anti-Bcl-xl, anti-PI3K class III,

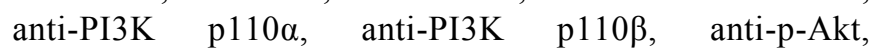
anti-p-PDK1, anti-GSK3- $\beta, \quad$ anti-p-c-Raf, anti p-NF- $\kappa$ B-p65, anti-p-ERK, anti-cyclin B1, anti-CDK4 and anti-CDK6. GAPDH was used as an internal control. After being stained with their respective secondary antibodies, the proteins were detected by Odyssey infrared imaging (LI-COR Biosciences, Lincoln, NE, USA).

\subsection{Statistical Analysis}

Statistical analyses were carried out by one-way ANOVA using SPSS statistical software version 16.0(SPSS, Inc., Chicago, IL, USA). All data are expressed as mean \pm SD. P-values $<0.05$ were considered to indicate a statistically significant result.

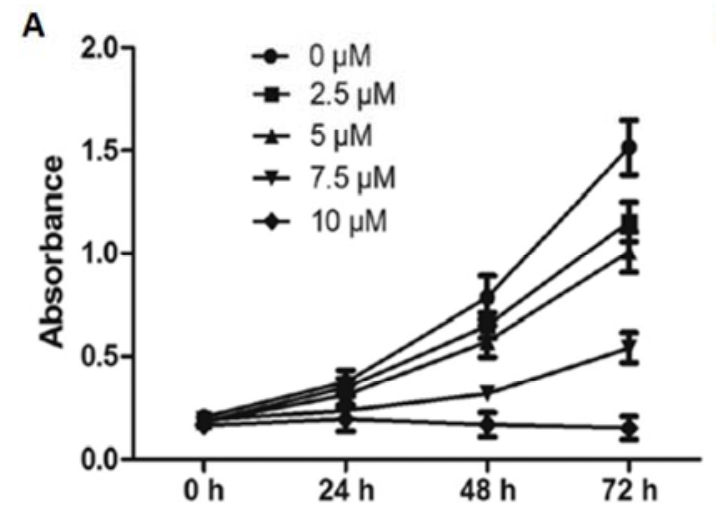

\section{Results}

\subsection{Effects of PEITC Treatment on the Viability of the NPC-TW01 and 16HBE Cell}

The influence of PEITC treatment on proliferation in human NPC-TW01 nasopharyngeal carcinoma cells and normal 16HBE bronchial epithelial cells was determined by CCK-8 assays. The results showed that PEITC exerted profound dose- and time-dependent antiproliferative effects on the growth of NPC-TW01 cells when administered between 0 and $10 \mu \mathrm{M}$ for treatment times from $0-72 \mathrm{~h}$ (Figure 1A). The inhibitory efficiency at $10 \mu \mathrm{M}$ PEITC in NPC-TW01 cells $24 \mathrm{~h}$ post-treatment exceeded $49 \%$. In contrast, the same treatment conditions had little effect on the proliferation of the 16HBE cells (Figure 1B). These results confirmed that NPC-TW01 tumor cells exhibited greater sensitivity to PEITC than normal 16HBE bronchial epithelial cells.

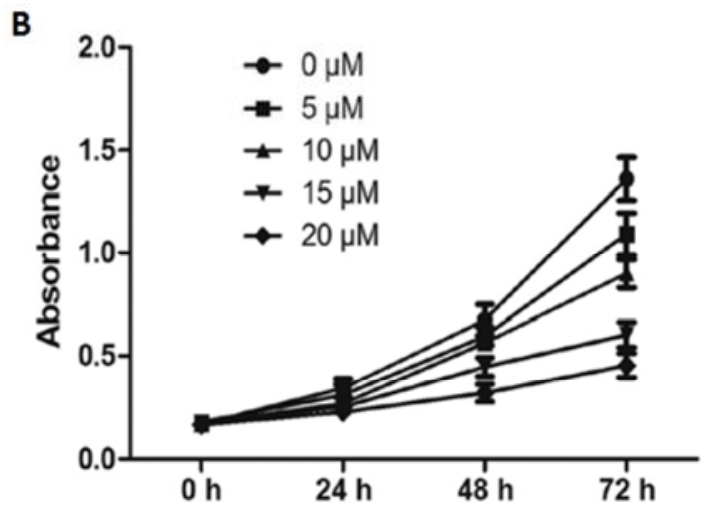

Figure 1. Viability of NPC-TW01 human nasopharyngeal carcinoma cells and 16HBE human bronchial epithelial cells following treatment with phenethyl isothiocyanate (PEITC). (A) The results from the CCK-8 assays showed that proliferation was significantly inhibited in the NPC-TW01 cells in a dose- and time-dependent manner. (B) However, proliferation was not significantly inhibited in the $16 H B E$ cells at concentrations $<10 \mu M$ PEITC and remained comparatively low up to a concentration of $20 \mu M$ PEITC. The data represent three independent experiments; error bars represent SD (ANOVA).

\subsection{Effects of PEITC on Apoptosis in the NPC-TW01 and $16 \mathrm{HBE}$ Cells}

To further explore the effects of PEITC treatment on nasopharyngeal carcinoma cells, the levels of apoptosis in the NPC-TW01 and 16HBE cells were determined by Annexin V-FITC/PI double-staining flow cytometry after treatment with various concentrations of PEITC for $24 \mathrm{~h}$. The results demonstrated that the percentages of both early (FITC+/PI-) and late apoptotic NPC-TW01 cells (FITC+/PI+) gradually increased with increasing concentrations of PEITC in a dose-dependent manner (Figure 2A). At $10 \mu \mathrm{M}$ PEITC, the percentages of apoptotic cells after $24 \mathrm{~h}$ treatment were 47.9 and $6.8 \%$ in the NPC-TW01 and 16HBE cells, respectively. In addition, the levels of apoptosis in the 16HBE cells remained comparatively low at higher concentrations of PEITC, with only 13.8 and $15.8 \%$ apoptotic cells at 15 and $20 \mu \mathrm{M}$ PEITC, respectively (Figure 2B). These results demonstrated that normal 16HBE bronchial cells exhibited higher tolerance to PEITC treatment than the NPC-TW01 tumor cells. In summary, PEITC appeared effective in inducing apoptosis in laryngeal cancer cells between 0 and $10 \mu \mathrm{M}$ PEITC in vitro while having little or no toxicological impact on normal non-tumorous bronchial cells.

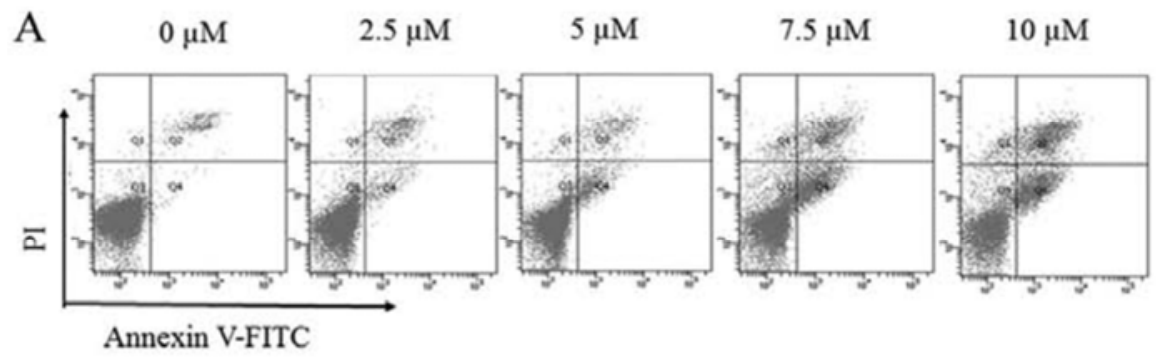


B

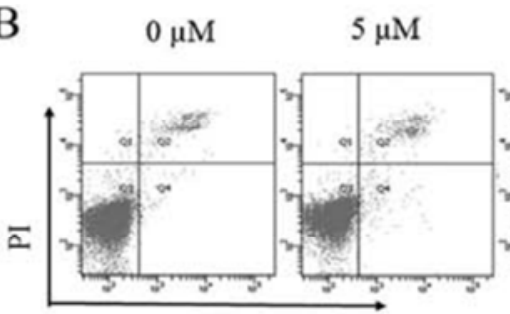

Annexin V-FITC

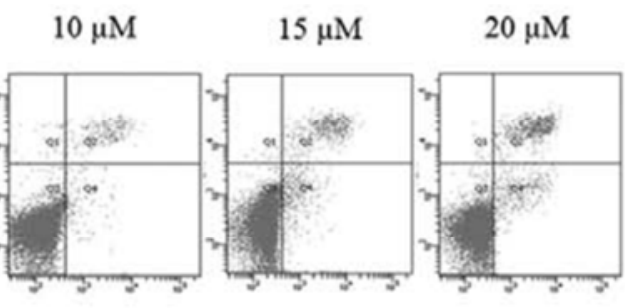

Figure 2. Annexin V-FITC flow cytometric analyses of apoptosis in NPC-TW01 and 16HBE cell lines treated with phenethyl isothiocyanate (PEITC). (A) The levels of both early (FITC+/PI-) and late apoptotic cells (FITC+/PI+) clearly increased in the NPC-TW01 cells with increasing concentrations of PEITC. (B) There was little change in the corresponding levels of apoptotic cells in the 16HBE cells. The percentages of apoptotic cells were significantly increased in NPC-TW01 cells at concentrations $\leq 10 \mu M$ PEITC in a dose-dependent manner; whereas there was little change in the percentages of $16 H B E$ cells up to $20 \mu M$ PEITC. The data represent three independent experiments; error bars represent SD (ANOVA).

\subsection{Effects of PEITC on Cell Cycle Arrest in the NPC-TW01Cell}

To investigate the effects of PEITC treatments on nasopharyngeal carcinoma in greater detail, flow cytometric analysis with PI single-staining was used to examine cell cycle distribution in the NPC-TW01 cells following a 24-h treatment with increasing concentrations of PEITC. As shown in Figure 3, the proportion of cells in the $\mathrm{G} 0 / \mathrm{G} 1$ phase decreased from 58.92 to $40.97 \%$ as PEITC $(\mathrm{P}<0.05)$ concentrations increased from $0-10 \mu \mathrm{M}$; whereas the corresponding proportion of $\mathrm{G} 2 / \mathrm{M}$ phase cells significantly increased from 7.01 to $23.35 \%$ ( $(\mathrm{P}<0.05)$; and the proportion of $\mathrm{S}$ phase cells increased marginally from 27.86 to $33.12 \%$. These results demonstrated that PEITC has the greatest influence in promoting cell cycle arrest in NPC-TW01 cells at the $\mathrm{G} 2 / \mathrm{M}$ phase.

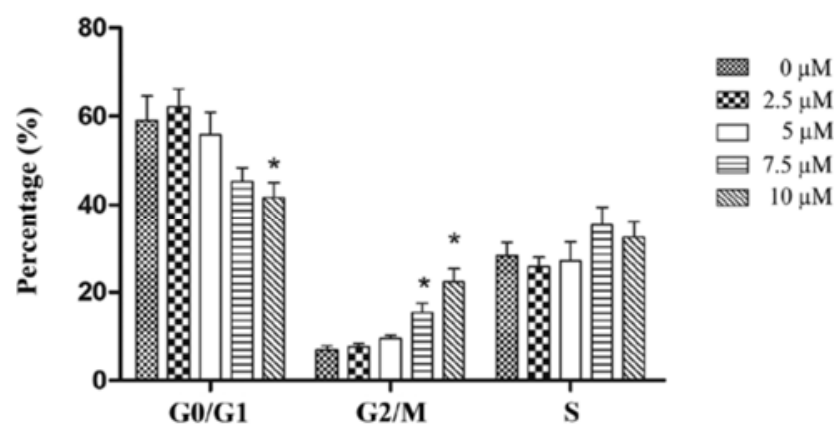

Figure 3. Flow cytometric analysis of cell cycle distribution in NPC-TW01 cell lines following treatment with PEITC. The proportion of cells in the G2/M phase increased in the NPC-TW01 cells as the concentration of PEITC increased. Significant concurrent decreases were noted in the percentages of cells in the G0/G1 phase and S phase while the percentages of G2/M phase cells increased during treatment. The data represent three independent experiments; error bars represent $S D(A N O V A)$. $* P<0.05$ compared to values at $0 \mu M$ PEITC.

\subsection{TUNEL Detection of Cell Apoptosis in the NPC-TW01 Cells}

TUNEL assays were performed to verify the flow cytometric results and to further explore the pro-apoptotic effects of PEITC treatment on the nasopharyngeal carcinoma cells. The micrographs were analyzed by fluorescence microscopy $24 \mathrm{~h}$ post-treatment and showed that the mean percentages of apoptotic increased from $1.29 \pm 2.78$ at a concentration of 0 PEITC to $4.83 \pm 1.85,9.02 \pm 4.37$, $20.05 \pm 1.73$ and $43.63 \pm 3.28 \%$ at concentrations of $2.5,5,7.5$ and $10 \mu \mathrm{M}$ PEITC, respectively. These results confirmed that PEITC induced apoptosis in the NPC-TW01 cells in a dose-dependent manner (Figure 4).

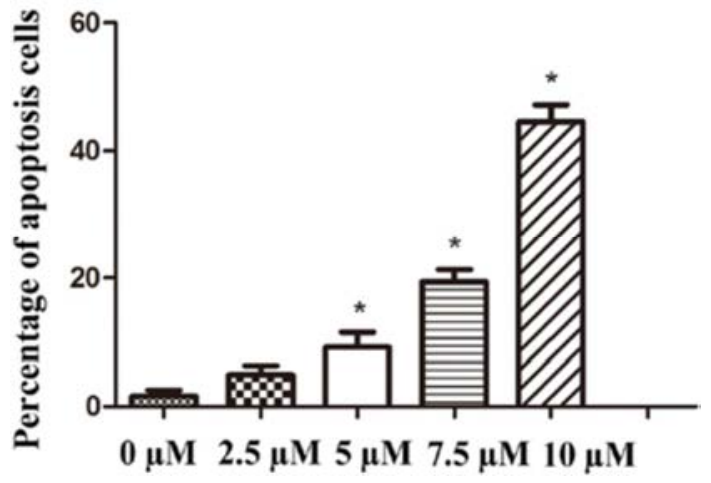

Figure 4. Detection of cell apoptosis in the NPC-TW01 nasopharyngeal carcinoma cells by TUNEL assays. Apoptosis in the NPC-TW01 cells increased as the concentrations of PEITC increased. Quantification of the TUNEL results indicate that the increases in apoptotic cells were significant at concentrations $>5 \mu M$ PEITC $24 \mathrm{~h}$ post-treatment. The data represent three independent experiments; error bars represent $S D$ (ANOVA). $* P<0.05$ relative to values at $0 \mu M$ PEITC.

\subsection{Effects of PEITC Treatment on Cell Invasion in the NPC-TW01 Cells}

A Transwell assay was performed to determine whether PEITC influences the invasiveness of NPC-TW01 nasopharyngeal carcinoma cells. Following treatment with $0-10 \mu \mathrm{M}$ PEITC for $48 \mathrm{~h}$, the data clearly demonstrated that PEITC suppressed the invasive ability of the NPC-TW01 cells in a dose-dependent manner (Figure 5). The effects were most significant at concentrations $\geq 5 \mu \mathrm{M}$ PEITC $(\mathrm{P}<0.05)$. 


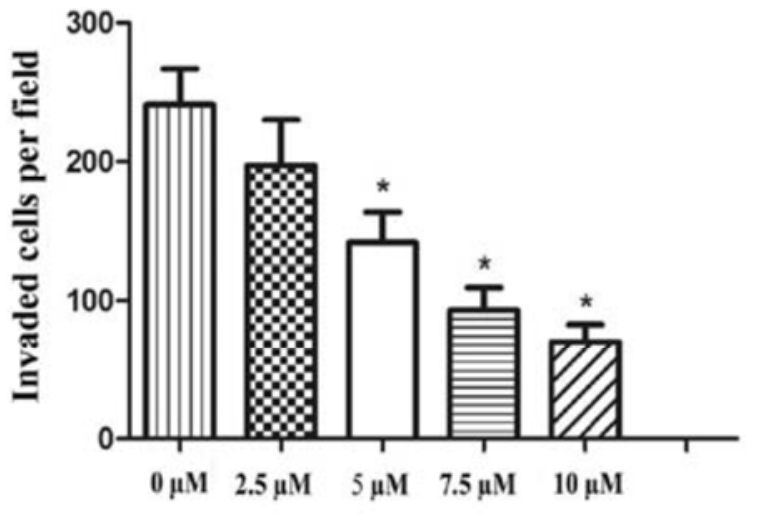

Figure 5. Transwell invasion assays of NPC-TW01 cells treated with PEITC. A decrease in the numbers of migrating NPC-TW01 cells was noted following a 48-h treatment with PEITC. Quantification of the cell counts at each concentration of PEITC are given in the plot, based on the mean values/field from at least five randomly selected low-power fields. The data represent three independent experiments; error bars represent $S D$ (ANOVA). $* P<0.05$ relative to values at $0 \mu M$ PEITC.

\subsection{Effects of PEITC Treatment on Protein Expression Levels Associated with Proliferation, Apoptosis and Cell Cycle Signaling Pathway}

Having demonstrated that PEITC treatment could have significant effects on proliferation, apoptosis, cell cycle distribution and invasion in NPC-TW01 cells, it was necessary to explore the potential mechanisms. Therefore, western blotting was performed to identify changes in the expression levels of regulatory proteins involved in key signaling pathways related to the occurrence and development of NPC. The pathways of interest included proliferation: PI3K, Akt, NF- $\mathrm{KB}$ and ERK; apoptosis: $\mathrm{Bcl}-2, \mathrm{Bcl}-\mathrm{xl}$ and $\mathrm{Bax}$; and cell cycle progression: GSK3- $\beta$, cyclin B1, CDK4 and CDK6. Qualitative analyses showed that as the PEITC concentration increased from 0 to 10 $\mu \mathrm{M}$, the expression levels of PI3K class III, PI3K p110 $\alpha$, PI3K

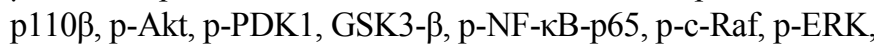
Bcl-2, Bcl-xl, cyclin B1, CDK4 and CDK6 were downregulated $24 \mathrm{~h}$ post-treatment and Bax was upregulated, whereas t-Akt, t-NF- $\kappa$ B-p65 and t-ERK protein expression remained unchanged as the PEITC concentration increased (Figure 6).

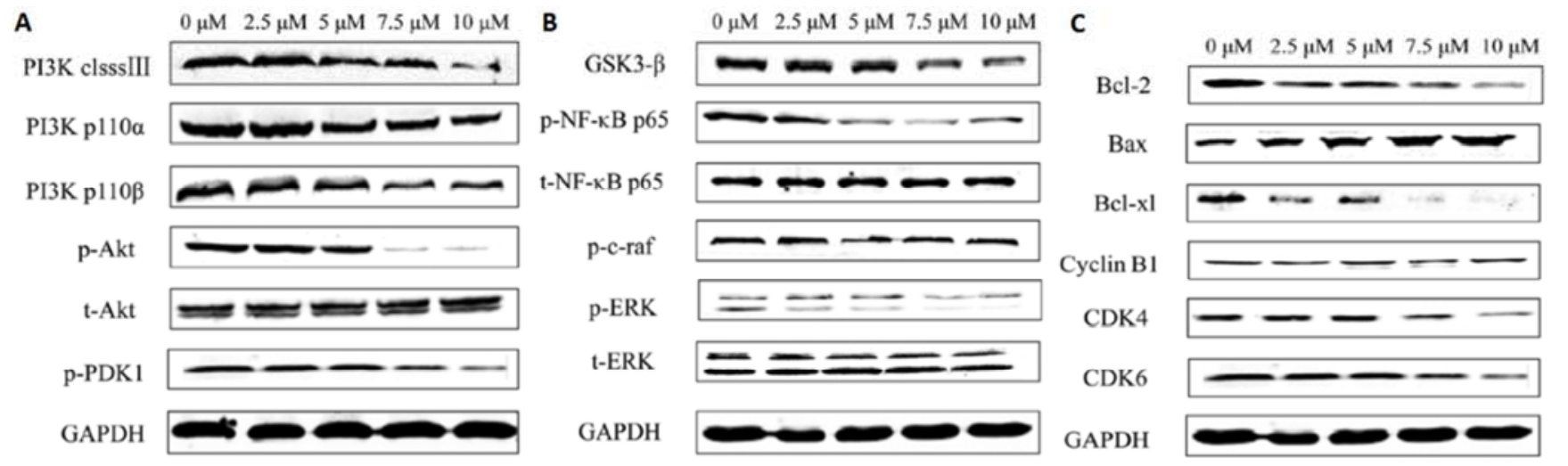

Figure 6. Western blot assays of protein expression levels in proliferation, apoptosis and cell cycle progression pathways in the NPC-TW01 cells treated with

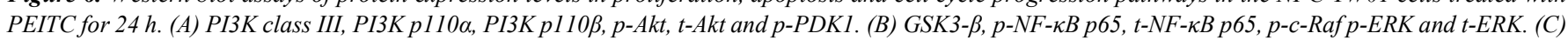
Bcl-2, Bax, Bcl-xl, cyclin B1, CDK4 and CDK6. Data presented are the means $\pm S D$. Results were normalized to GAPDH.

\section{Discussion}

It has been reported that the median survival time for an NPC patient with metastatic or advanced disease was only 5 to 11 months [13]. Although NPC is a radiosensitive tumor compared with other head or neck cancers, the high incidence of recurrence, lymph node spread and distant metastases cause poor prognosis [14]. Chemotherapy plays an important role in the treatment of NPC. The major problem of current chemotherapy is the high toxicity of the drugs being used [15]. Therefore, NPC chemotherapy needs drugs with improved efficacy and safety.

PEITC is an active ingredient extracted from natural cruciferous plants. It shows significant anti-tumor effect in various tumor cells. However, its actions in nasopharyngeal carcinoma, remain largely unknown. Therefore the purpose of the present study was to explore its actions and potential mechanisms in human NPC-TW01 nasopharyngeal carcinoma cells and normal 16HBE bronchial epithelial cells.
Our study showed that treatment with PEITC significantly suppressed proliferation, induced apoptosis, promoted G2/M cell cycle arrest and inhibited invasion and thereby metastasis, in the NPC-TW01 cancer cells in vitro $(\mathrm{P}<0.05)$. Importantly, the results also demonstrated that treatment within a safe range of concentrations (from 0-10 $\mu \mathrm{M}$ PEITC) had little toxicological impact on normal 16HBE cells. These findings were consistent with published studies on other types of cancer [16]; for example, Wang et al showed that treat- ment with 5-10 $\mu \mathrm{M}$ PEITC for 8-24 h could inhibit proliferation and induce apoptosis in cervical cancer cells [17].

Our results also showed that PEITC significantly inhibited proliferation in NPC-TW01 cells in a time- and dose-dependent manner, and significantly promoted apoptosis and inhibited cell invasion in a dose-dependent manner $(\mathrm{P}<0.05)$. Similar observations have been reported in previous studies $[18,19]$. For example, the percentage of NPC-TW01 cells was reduced to $49 \%$ and the percentage of apoptotic cells was increased to $46 \% 24 \mathrm{~h}$ post-treatment with $10 \mu \mathrm{M}$ PEITC relative to the corresponding levels at $0 \mu \mathrm{M}$ PEITC $(\mathrm{P}<0.05)$. 
These effects were accompanied by concurrent increases in the percentages of cells at the $\mathrm{G} 2 / \mathrm{M}$ phase, suggesting that PEITC may inhibit cell growth by inducing cell cycle arrest at the $\mathrm{G} 2 / \mathrm{M}$ checkpoint.

Tumorigenesis is characterized by uncontrolled cell growth and tumor formation and is associated with alterations in the expression levels of proteins involved in pro-survival signaling pathways linked to the occurrence and development of tumors [20]. Furthermore, the suppression of multiple signaling pathways simultaneously has been found to have a greater anticancer impact than suppression of a single signaling pathway alone [21]. Proteins such as PI3K, Akt, ERK and NF- $\kappa \mathrm{B}$ have been found to play key roles in the regulation of proliferation, cell cycle progression and apoptosis in NPC [22]. RNAi is frequently used to inhibit the expression of proteins and has proven to be important in developing effective therapeutic strategies against cancers. Using these methods, our results indicated that PEITC may target several important signaling pathways associated with apoptosis, cell growth and metastasis simultaneously, thereby enhancing its antitumor impact on nasopharyngeal carcinoma.

Metastasis and tumor cell invasion are important factors in the prognosis and recurrence of cancers. Our results showed that treatment with PEITC inhibited the invasiveness of NPC-TW01 cells in vitro and suggested that this effect was due in part to the suppression of ERK and NF- $\kappa B$ activity. Similar findings were reported by Gupta et al in a mouse model of breast cancer in which the administration of PEITC suppressed development of metastasized tumors [23]. However, data on the antimetastatic effects of PEITC in other forms of cancer remain scarce, and further investigations is needed to elucidate the molecular mechanisms.

Nowadays, radiotherapy or/and chemotherapy is the primary treatment for NPC. Although NPC is a radiosensitive tumor compared with other head or neck cancers, the high incidence of recurrence, lymph node spread and distant metastases cause poor prognosis Consequently, there is increasing interest in identifying natural compounds with effective anticancer properties due their low toxicities [24]. The results of the present study demonstrated that PEITC was effective in suppressing proliferation, invasion and inducing apoptosis in NPC-TW01 nasopharyngeal carcinoma cells while having little toxicological impact on $16 \mathrm{HBE}$ normal bronchial epithelial cells, particularly within a range of 0-10 $\mu \mathrm{M}$ PEITC. Although, increased levels of apoptosis were observed in 16HBE cells at higher concentrations of PEITC (up to $20 \mu \mathrm{M}$ ), these remained comparatively low relative to the levels in the NPC-TW01 cells. Similar observations have been reported previously. PEITC was found to inhibit mammary carcinoma tissues in a mouse model, but was well-tolerated in normal mammary glands [25]; however, another study reported increased levels of aspartate aminotransferase (AST) at high doses of PEITC (100-150 $\mathrm{mg} / \mathrm{kg}$ ) but no change was observed at lower doses $(50 \mathrm{mg} / \mathrm{kg})$, suggesting that PEITC may cause dose-dependent toxicity in normal tissues at high treatment concentrations [26].
Variations in treatment times or analytical time points between different studies may have contributed to these inconsistencies [27]. Therefore, further in vivo studies are required to define the toxicity profile of PEITC in nasopharyngeal carcinoma in order to ensure that doses can be titrated accurately in a clinical setting.

\section{Conclusion}

In summary, the study demonstrated that PEITC significantly induced antiproliferative, pro-apoptotic and antimetastatic effects in NPC-TW01 human nasopharyngeal carcinoma cells in a time- and dose-dependent manner, while presenting little toxicological damage to normal 16HBE bronchial epithelial cells in vitro. The results suggest that its anticancer activities resulted from its ability to inhibit several critical pro-survival pathways related to the occurrence and development of nasopharyngeal carcinoma simultaneously by dysregulation of key cell signaling proteins. In summary, PEITC may offer a valuable contribution to the development of novel therapeutic strategies for the treatment of NPC in the future.

\section{Acknowledgements}

Author Cheng-Gang Mao and Author Xiao-Chun Zhou both contributed equally to this work.

This study was supported by grants from the Science and Technology Program of Jingzhou City (nos. 2017038; 2017044).

\section{References}

[1] Brennan B: Nasopharyngeal carcinoma. Orphanet J Rare Dis 1: 23, 2006.

[2] Afqir S, Ismaili $\mathrm{N}$ and Errihani $\mathrm{H}$ : Concurrent chemoradiotherapy in the management of advanced nasopharyngeal carcinoma: current status. J Cancer Res Ther 5: 3-7, 2009.

[3] Razak AR, Siu LL, Liu FF, Ito E, O'Sullivan B and Chan K: Nasopharyngeal carcinoma: the next challenges. Eur J Cancer 46: 1967-1978, 2010.

[4] Zhou JX, Han JB, Chen SM, Xu Y, Kong YG, Xiao BK and Tao ZZ: $\gamma$-secretase inhibition combined with cisplatin enhances apoptosis of nasopharyngeal carcinoma cells. Exp Ther Med 3: 357-361, 2012.

[5] Kolonel LN, Hankin JH, Whittemore AS, Wu AH, Gallagher RP, Wilkens LR, John EM, Howe GR, Dreon DM, West DW, et al: Vegetables, fruits, legumes and prostate cancer: A multiethnic case-control study. Cancer Epidemiol Biomarkers Prev 9: 795-804, 2000.

[6] Hanahan D and Weinberg RA: Hallmarks of cancer: The next generation. Cell 144: 646-674, 2011.

[7] Annema N, Heyworth JS, McNaughton SA, Iacopetta B and Fritschi L: Fruit and vegetable consumption and the risk of proximal colon, distal colon, and rectal cancers in a case-control study in Western Australia. J Am Diet Assoc 111: 1479-1490, 2011. 
[8] Gupta P, Kim B, Kim SH and Srivastava SK: Molecular targets of isothiocyanates in cancer: Recent advances. Mol Nutr Food Res 58: 1685-1707, 2014.

[9] Trachootham D, Zhou Y, Zhang H, Demizu Y, Chen Z, Pelicano H, Chiao PJ, Achanta G, Arlinghaus RB, Liu J, et al: Selective killing of oncogenically transformed cells through a ROS-mediated mechanism by beta-phenylethyl isothiocyanate. Cancer Cell 10: 241-252, 2006.

[10] Huong D, Shim JH, Choi KH, Shin JA, Choi ES, Kim HS, Lee SJ, Kim SJ, Cho NP and Cho SD: Effect of $\beta$-phenylethyl isothiocyanate from cruciferous vegetables on growth inhibition and apoptosis of cervical cancer cells through the induction of death receptors 4 and 5. J Agric Food Chem 59: 8124-8131, 2011.

[11] Sakao K, Desineni S, Hahm ER and Singh SV: Phenethyl isothiocyanate suppresses inhibitor of apoptosis family protein expression in prostate cancer cells in culture and in vivo. Prostate 72: 1104-1116, 2012.

[12] Chan ATC: Nasopharyngeal carcinoma. Ann Oncol 21 (Suppl 7): vii308-vii312, 2010.

[13] Teo PM, Kwan WH, Lee WY, Leung SF, Johnson PJ (1996) Prognosticators determining survival subsequent to distant metastasis from nasopharyngeal carcinoma. Cancer 77: 2423 2431.

[14] Sham JS, Choy D, Choi PH (1990) Nasopharyngeal carcinoma: the significance of neck node involvement in relation to the pattern of distant failure. Br J Radiol 63: 108-113.

[15] Ma BB, Chan AT (2005) Recent perspectives in the role of chemotherapy in the management of advanced nasopharyngeal carcinoma. Cancer 103: 22-31.

[16] Huong LD, Shin JA, Choi ES, Cho NP, Kim HM, Leem DH and Cho SD: $\beta$-Phenethyl isothiocyanate induces death receptor 5 to induce apoptosis in human oral cancer cells via p38. Oral Dis 18: 513-519, 2012.

[17] Wang XF, Wu DM, Li BX, Lu YJ and Yang BF: Synergistic inhibitory effect of sulforaphane and 5-fluorouracil in high and low metastasis cell lines of salivary gland adenoid cystic carcinoma. Phytother Res 23: 303-307, 2009.
[18] Gupta P and Srivastava SK: Antitumor activity of phenethyl isothiocyanate in HER2-positive breast cancer models. BMC Med 10: 80, 2012.

[19] Chen G, Chen Z, Hu Y and Huang P: Inhibition of mitochondrial respiration and rapid depletion of mitochondrial glutathione by $\beta$-phenethyl isothiocyanate: Mechanisms for anti-leukemia activity. Antioxid Redox Signal 15: 2911-2921, 2011.

[20] Doerfler W, Hohlweg U, Müller K, Remus R, Heller H and Hertz J: Foreign DNA integration - perturbations of the genome - oncogenesis. Ann NY AcadSci 945:276-288, 2001.

[21] Liu SF, Wang H, Lin XC, Xiang H, Deng XY, Li W, Tang M and Cao Y: NF-kappaB inhibitors induce lytic cytotoxicity in Epstein-Barr virus-positive nasopharyngeal carcinoma cells. Cell Biol Int 32: 1006-1013, 2008.

[22] Wang YQ, Chen C, Chen Z, Xu Y, Wang Y, Xiao BK, Chen SM and Tao ZZ: Indole-3-carbinol inhibits cell proliferation and induces apoptosis in Hep-2 laryngeal cancer cells. Oncol Rep 30: 227-233, 2013.

[23] Gupta P, Adkins C, Lockman P and Srivastava SK: Metastasis of breast tumor cells to brain is suppressed by phenethyl isothiocyanate in a novel in vivo metastasis model. PLoS One 8: e67278, 2013.

[24] Newman DJ and Cragg GM: Natural products as sources of new drugs over the 30 years from 1981 to 2010 . J Nat Prod 75: 311-335, 2012.

[25] Singh SV, Kim SH, Sehrawat A, Arlotti JA, Hahm ER, Sakao K, Beumer JH, Jankowitz RC, Chandra-Kuntal K, Lee J, et al: Biomarkers of phenethyl isothiocyanate-mediated mammary cancer chemoprevention in a clinically relevant mouse model. J Natl Cancer Inst 104: 1228-1239, 2012.

[26] Manesh C and Kuttan G: Effect of naturally occurring isothiocyanates on the immune system. Immunopharmacol Immunotoxicol 25: 451-459, 2003.

[27] Wang LG and Chiao JW: Prostate cancer chemopreventive activity of phenethyl isothiocyanate through epigenetic regulation (Review). Int J Oncol 37: 533-539, 2010. 